\title{
Inschriftenkulturen im kommunalen Italien. Ergebnisse und Perspektiven
}

Wie ausführlich von Marc von der Höh in der Einleitung zu diesem Sammelband ausgeführt, folgen die hier versammelten Aufsätze in Anlehnung an die methodische Konzeption des SFB 933 „Materiale Textkulturen“ einem innovativen, kulturwissenschaftlich geprägten Zugriff auf die mittelalterliche Inschriftenkultur: Sie stellen weniger den Text der ,Inschrift‘ in das Zentrum ihrer Überlegungen als vielmehr das Artefakt selbst in seiner komplexen materialen Gestalt, seinem Verhältnis zum Raum, seinen historischen und sozialen Kontexten und seiner Wahrnehmung und Funktionalisierung durch sich wandelnde Rezipientenkreise. Ein solcher Ansatz kann nur bei sorgfältiger und differenzierter Analyse der Schriftformen, des Trägermaterials, der Anbringungssituationen und der verschiedenen, auf unterschiedlichste Weise mit den Inschriften interagierenden Personen und Personengruppen zielführend sein. In welcher Weise er von den Autorinnen und Autoren umgesetzt wurde, mag zunächst ein knapper, resümierender Überblick über die einzelnen Beiträge zeigen.

\section{Zehn Schlaglichter auf das Thema}

Trotz ihrer beeindruckenden thematischen Breite und Multiperspektivität lassen sich zwischen den Aufsätzen dieses Sammelbandes mit aller gebotenen Vorsicht miteinander verwandte Fragestellungen und Zugänge identifizieren, die eine Gruppierung der Beiträge in drei Blöcken nahegelegt haben. So richten die ersten vier Artikel ein besonderes Augenmerk auf die praxeologische Dimension mittelalterlicher Inschriftlichkeit. Marialuisa Bottazzi untersucht dabei Kontinuitäten und Umbrüche in der Entwicklung der Inschriftenkultur(en) auf der Apenninenhalbinsel zwischen Spätantike und früher Neuzeit und setzt ihre Befunde zu neuen Erkenntnissen aus den letzten 30 Jahren internationaler epigraphischer Forschung in Beziehung. Zwar könne man vereinzelt auf Belege für die Verwendung von Epigraphik durch Laien auch in den Jahrhunderten des frühen Mittelalters stoßen, doch sei an der kirchlichen Dominanz auf diesem Felde nicht zu zweifeln. Der Pontifikat Bischof Damasus' I. wird von ihr als eine wesentliche Umbruchzeit in der Geschichte der spätantik-frühmittelalterlichen Epigraphik identifiziert, weil durch Damasus ein eigener Schrifttypus von hoher graphischer Qualität eingeführt und neue Praktiken für die Nutzung von

Dieser Beitrag ist im Heidelberger Sonderforschungsbereich 933 „Materiale Textkulturen. Materialität und Präsenz des Geschriebenen in non-typographischen Gesellschaften“ entstanden. Der SFB 933 wird durch die Deutsche Forschungsgemeinschaft finanziert.

ฮ open Access. ( 2019 Nikolas Jaspert, Christian Witschel, publiziert von De Gruyter. (c) BY-NC-ND Dieses Werk ist lizenziert unter der Creative Commons Attribution-NonCommercial-NoDerivatives 4.0 Lizenz. 
Inschriften durch die römische Kirche etabliert wurden. Inschriftensammlungen des frühen Mittelalters tradierten dieses Wissen um ältere Formen der Epigraphik an spätere Generationen; als herausragendes Beispiel hierfür wird der Liber Pontificalis Ecclesiae Ravennatis des Agnellus von Ravenna aus dem 9. Jahrhundert ausführlicher vorgestellt. Funeräre Kontexte bildeten eine weitere Grundlage für gewisse Kontinuitäten, wenngleich an einer massiven Abnahme der epigraphischen Produktion insbesondere zwischen dem 6. und 8. Jahrhundert letztlich nicht zu zweifeln sei.

Nicoletta Giovè schreitet ein breites Spektrum öffentlich präsentierter, materialer Träger von Texten im städtischen Raum Mittel- und Norditaliens ab. Solche „scritture esposte“ (,ausgestellte Schriften`) wurden vorwiegend an neuralgischen Knotenpunkten des städtischen Verkehrs platziert. Das Panorama der Anbringungsorte reicht von Brunnen und Stadttoren bis hin zu Türmen und Straßenkreuzungen. Dezidiert ordnet Giovè diese beschrifteten Artefakte einem neuen Selbstverständnis laikaler städtischer Führungsschichten zu, die - teilweise im Rückgriff auf antike Vorbilder - seit dem 12. Jahrhundert die Kommunikation mit der städtischen Öffentlichkeit und auswärtigen Besuchern mithilfe dieses ,wiederentdeckten` Mediums anstrebten. Dabei folgt Giovè der Prämisse, dass ,ausgestellte‘ Inschriften in der Zeit der Kommunen ein eigenes System bildeten, welches nicht unbedingt auf die Lektüre der Inschriftentexte, sondern vor allem auf ihre markante Sichtbarkeit ausgerichtet war. Sie sprachen unterschiedliche Zeitebenen an und konnten daher mehrere Funktionen zugleich erfüllen: Kommunale Inschriften kommemorierten vergangene Ereignisse, ebenso wie sie prospektiv zum Gedenken oder im Sinne der Didaxe zum Handeln aufriefen. In diesem Sinn repräsentierten sie im Zeitraum zwischen dem 11. und 13. Jahrhundert das spezifische Selbstverständnis aufstrebender städtischer Führungsgruppen und Korporationen in den italienischen Städten.

Vincent Debiais widmet sich den sog. ,Urkundeninschriften“ (,chartes lapidaires“), also Inschriften, die in formaler und/oder inhaltlicher Hinsicht mittelalterlichen Urkunden ähneln. In einer beeindruckenden Tour d'Horizon europäischer Urkundeninschriften des hohen und späten Mittelalters kann der Autor zeigen, dass es sich bei diesen Artefakten keineswegs um schlichte Übertragungen von Schriftstücken in ein anderes, dauerhaftes Material handelte. Vielmehr wiesen unter anderem Layout und Gestaltung solcher Inschriften visuelle Eigenheiten eines besonders herausgehobenen Gegenstands von hoher graphischer Qualität auf, der sowohl die Erinnerung an die Umstände seiner Entstehung als auch Hinweise auf zukünftige performative Handlungen, zu denen der in Stein gemeißelte Text gleichsam aufrief, in sich trug. Die „chartes lapidaires“ erlangten vor diesem Hintergrund vor allem einen eminent öffentlichen Charakter, denn sie waren sowohl Dokument als auch Monument einer städtischen Inszenierung. Debiais' Analysen unterstreichen zudem die von Marc von der Höh am Beispiel Modenas illustrierte Beobachtung, wonach die Zeitgenossen des 11.-13. Jahrhunderts durchaus eine Vorstellung von der Historizität von Schrift und der Zeitgebundenheit bestimmter paläographischer Merkmale besaßen. 
Flavia De Rubeis entwickelt eine Typologie öffentlicher Inschriftlichkeit im kommunalen Zeitalter. Dazu differenziert sie in einem ersten Schritt nach dem Anbringungsort der Inschriften, nach deren Schriftgestalt sowie Inhalt. In diachroner Perspektive kann sie eine gewisse (wenngleich keine allumfassende) „ecclesializzazione“ der Epigraphik auf der Apenninenhalbinsel zwischen dem 6. und 11. Jahrhundert feststellen, die sich auch im Schriftbild - generell in einer Abnahme der Monumentalität - niederschlug. In einem zweiten Schritt korreliert sie die Auftraggeber einer Inschrift mit deren intendierter Funktion. Dazu unterscheidet sie drei Absichten, die Inschriften im öffentlichen Raum erfüllen sollten: Die Stiftung kollektiver Erinnerung an einen Rechtsakt, die Erinnerung an ein Ereignis oder die Erhöhung einer Person bzw. Gruppe. Ihre aufschlussreichen, bislang nicht sonderlich prominent in der Literatur behandelten Beispiele ermöglichen anregende Vergleiche insbesondere zu den Aufsätzen von Rebecca Müller und Henrike Haug. Die untersuchten Artefakte zeigen außerdem, dass es für deren Gestaltung letztlich nicht wesentlich war, ob eine Institution bzw. eine Gemeinschaft oder ein Individuum als Auftraggeber fungierten. Entscheidend war vielmehr, ob eine Inschrift vorwiegend einen dokumentierenden (und damit im Kern einen rechtlichen) oder einen kommemorierenden (und damit vor allem Identität stiftenden) Charakter aufwies, was sich tendenziell in einer erhöhten Lesbarkeit des zweiten Typus niederschlug.

Eine zweite, insgesamt drei Texte umfassende Gruppe von Aufsätzen fokussiert in besonderem Maße die Präsenz von epigraphischen Monumenten im öffentlichen Raum. Wilfried E. Keil analysiert das Verhältnis zwischen Skulpturen und Inschriften an drei Orten in Nord- und Mittelitalien - Genua, Mailand und Montefalco. Der Vergleich zwischen diesen Beispielen zeigt, dass in einigen Fällen Schrift der Verdeutlichung des Bildwerks diente, während sie diesem in anderen Fällen untergeordnet war oder sogar dezidiert zu einem späteren Zeitpunkt ,veruneindeutigt' wurde. Im Genueser Kommunalpalast zum Beispiel verschob man eine Inschrift durch die Anbringung eines erbeuteten Löwenkopfs und schränkte dabei ihre Lesbarkeit ein, womit die Erinnerung an einen in Ungnade gefallenen Stadtherren verdeckt, aber nicht ausgelöscht wurde. Das Beispiel illustriert schlagend, dass nachträglich und aktiv die Schriftpräsenz kommunaler Inschriften eingeschränkt werden konnte (,restringierte Schriftpräsenz'). Zugleich diente der nunmehr um eine Spolie ergänzte Inschriftenträger als Trophäe und rief den Baumeister des Palastes in Erinnerung. Das Reiterstandbild des Mailänder Podestà Odalricus von 1233 hingegen war auf dessen Memoria ausgerichtet, während ein nur schwer lesbares Artefakt am Stadttor von Montefalco vor allem durch sein prominentes Wappen auf den Besucher der Ortschaft wirkte.

Rebecca Müller liefert einen breiten Überblick über die schrifttragenden Artefakte (dingliche Trophäen, Reliefs, Siegel, Wappen, Inschriften) in Genua während des 12. bis 14. Jahrhunderts. Erinnerungsstücke an militärische Siege über konkurrierende muslimische und christliche Mächte wurden ostentativ im öffentlichen Raum präsentiert und mit monumentalen Inschriften versehen. Die Verfasserin kann 
wahrscheinlich machen, dass die Führungsgruppen der Stadt auf der Grundlage älterer Praktiken somit einen markanten ,epigraphic habit' entwickelten, der durch die Verbindung von Bild und Text - also durch die „Bildlichkeit des dreidimensionalen Dings“ - gekennzeichnet war. Ihre sorgfältige Analyse herausragender Artefakte zeigt, dass Schrift im Einzelfall Lücken einer visuellen Botschaft füllen, in anderen Fällen deren Inhalt verstärken konnte. Monumentalinschriften an den Kirchen San Matteo oder San Lorenzo zu Genua zum Beispiel dienten als ,aide mémoire‘ herausragender Ereignisse der kommunalen Geschichte, aber auch imaginierter und dennoch im kollektiven Bewusstsein tief verankerter Geschehnisse wie der mythischen Stadtgründung durch den legendären Janus. Der Beitrag liefert damit eine Fallstudie für die Multifunktionalität städtischer Epigraphik, die in den Beiträgen von Flavia De Rubeis und Nicoletta Giovè ebenfalls prominent zutage tritt.

Henrike Haug wählt einen plurimedialen Zugang, um sich der Erinnerungskultur in den Städten Norditaliens und der Funktionalisierung von epigraphischen Zeugnissen in urbanen Kontexten zu nähern. Die Eliten Genuas und Pisas feierten militärische Erfolge und andere, für die Kommune als bedeutsam wahrgenommene und kommunizierte Ereignisse in unterschiedlichen Medien: In der Chronistik, in Fresken, aber eben auch in Inschriften. Damit schufen und perpetuierten sie das Selbstbild einer verfassten und wehrhaften Einung der Stadtbürger. Die Verfasserin unternimmt den Versuch, Dialoge und damit Interdependenzen zwischen Inschriften und Historiographie zu identifizieren. In der Tat weisen die Chroniken Genuas und Pisas so klare inhaltliche Ähnlichkeiten zu prominent im öffentlichen Raum, etwa an Stadttoren, angebrachten Inschriften auf, dass Haug mit Recht von einer ,intermedialen städtischen Erinnerungskultur“ sprechen kann. Im Falle Pisas lässt sich zudem beobachten, dass einige Ereignisse vorzugsweise in epigraphischer Form, andere hingegen historiographisch ausgeschmückt wurden. Eine Brücke zum Beitrag von Vincent Debiais wird schließlich dadurch geschlagen, dass Frau Haug mit dem im Codex Vetustior kopierten Wortlaut und Schriftbild einer Jerusalemer Inschrift ebenfalls den Medienwechsel eines Textes vorführt - hier jedoch nicht von der Handschrift zur Inschrift, sondern von der Inschrift zur Handschrift.

Die dritte und letzte Gruppe von Aufsätzen analysiert insbesondere die Wahrnehmung und Nutzung antiker Inschriften während des Mittelalters, die bereits in der Einleitung als ein zentraler Bestandteil der epigraphischen Kultur der Kommunen des Hochmittelalters angesprochen worden ist. Arnold Esch fragt nach den Gründen für die Verwendung antiker Spolien in mittelalterlichen Kirchen: War es deren Textgehalt, deren ästhetischer Reiz, deren Alter oder die mit ihnen und der Antike verbundenen Assoziationen? Eine umfassende Antwort auf diese Frage ist nicht möglich, wie eine Betrachtung größerer und kleinerer Orte in Italien deutlich macht. Während die Führungsgruppen der Stadt Pisa durch den Import römischer Spolien unmissverständlich einen Bezug zwischen ihrer Stadt und der Metropole Rom zum Ausdruck bringen wollten, dürfte die Nutzung antiker Inschriften in Landkirchen pragmatischeren, vielleicht auch ästhetischen Gründen geschuldet gewesen sein. Die Lesbarkeit der 
Artefakte war dabei wohl nicht das Entscheidende, sondern ihre Altehrwürdigkeit „nicht lesen sollte man, sondern staunen“. Die Verbauung römischer Meilensteine im Mittelalter wiederum könnte als vornehmlich pragmatisch motivierte Verwertung von Baumaterial verstanden werden, doch bot sie dem Bauherrn auch die Gelegenheit, die Heranschaffung von Gegenständen aus weiter Entfernung ostentativ zum Ausdruck zu bringen und zu zelebrieren. Auch in der Frage der Spolienverwendung gilt es daher, soweit möglich in jedem Einzelfall historische Kontexte zu rekonstruieren und unterschiedliche Kausalitäten in Rechnung zu stellen.

Katharina Bolle konstatiert nicht nur die quantitative und qualitative Diskrepanz zwischen den ,civic inscriptions' des antiken und des mittelalterlichen Rom, sondern fragt auch nach dem handwerklichen und intellektuellen Rüstzeug, das den Auftraggebern und Herstellern von Inschriften im hohen Mittelalter zur Verfügung stand. Damit greift sie konsequent die in der Einleitung zu diesem Band von Marc von der Höh formulierte Unterscheidung zwischen ,Wissen' und ,Anschauung‘ auf. Aus der kommunalen Epoche der Stadt Rom liegen einige Hinweise auf die Wahrnehmung antiker Texte vor, deren Lektüre aber den mittelalterlichen Zeitgenossen offenbar große Schwierigkeiten bereitete; von einem regelrechten Diskurs über Inschriftlichkeit könne somit keine Rede sein. Dennoch belegen einige Metatexte wie die Mirabilia Romae die Kenntnisnahme und absichtsvolle Verwendung antiker Inschriften, aber auch die Begrenztheit solcher Praktiken, denn viele prominente antike Inschriften werden hier nicht erwähnt. Einzelne herausragende epigraphische Produkte des 12. Jahrhunderts zeigen die bewusste Anlehnung an die Antike vor dem Hintergrund zeitgenössischer Transformationsprozesse, in deren Rahmen enge terminologische und institutionelle Anlehnungen an das republikanische Rom gesucht wurden. Im Vergleich mit anderen Zentren der kommunalen Bewegung könne man für Rom sogar einen spezifischen ,epigraphic habit' konstatieren. Die Wiederverwendung älterer und die Herstellung neuer Artefakte von hoher graphischer Qualität an der Casa dei Crescenzi zum Beispiel illustriert das Zeitgefühl eines Umbruchs, das sich in einer prononcierten Rückbesinnung auf den Glanz des alten Rom manifestierte.

Erik Beck und Lukas Clemens untersuchen die Wahrnehmung und Nutzung römischer Inschriften in Frankreich und im Römisch-Deutschen Reich des 10.-15. Jahrhunderts, indem sie Erwähnungen antiker epigraphischer Monumente in mittelalterlichen Texten nachspüren. Mit der Vita sancti Heriberti und dem Werk De incendio des Rupert von Deutz sowie einer Wegbeschreibung des 13. Jahrhunderts stellen sie Metatexte vor, die davon Zeugnis ablegen, wie Inschriften der Vergangenheit zur Erklärung mittelalterlicher Gegenwarten benutzt wurden. Auch in anderen Fällen wurde die - vermeintliche oder tatsächliche - imperiale Geschichte einzelner Orte an antiken Artefakten festgemacht. Ähnlich den von Rebecca Müller und Henrike Haug untersuchten genuesischen Eliten nutzten auch die Führungsgruppen in Konstanz und Salzburg im Hochmittelalter Informationen aus römischen Inschriften für die Konstruktion einer städtischen Eigengeschichte. Ein in St. Eucharius/St. Matthias zu Trier aufgefundener, aus einer wiederverwendeten und im 11. Jahrhundert ergänzten 
Inschriftenplatte geformter Blockaltar wiederum belegt die mittelalterliche Auseinandersetzung mit antiken Schriftformen. Das aussagekräftigste Fallbeispiel des Beitrags sind jedoch die römischen Inschriften aus Baden-Baden, die kundigen Fälschern des frühen 12. Jahrhunderts als Grundlage dienten, um ihren Besitzansprüchen ein vermeintlich hohes Alter und damit ein besonderes Gewicht zu verleihen.

\section{Voraussetzungen: Die Inschriftenkultur der Spätantike}

Bereits mehrfach ist in der Zusammenfassung der einzelnen Beiträge des Bandes die Bedeutung antiker epigraphischer Zeugnisse für die mittelalterliche Inschriftenkultur in Italien angesprochen worden. Die Betrachtung der ,longue durée' bei der Entwicklung der epigraphischen Praxis im Mittelmeerraum zwischen Antike und Mittelalter ist auch ein zentraler Bestandteil der Forschungsarbeit des Teilprojekts A01 innerhalb des SFB 933, aus dem dieser Band in Zusammenarbeit mit Marc von der Höh und der Universität Rostock hervorgegangen ist. Eine solche Perspektive soll daher an dieser Stelle aus althistorischer Perspektive noch etwas weiter vertieft werden. Hierbei steht insbesondere die Epoche der Spätantike als Zeit der Transformation zwischen Antike und Mittelalter - auch in Bezug auf die Verwendung von Inschriften - im Fokus. In regionaler Hinsicht soll der Schwerpunkt auf Nord- und Mittelitalien liegen.

Zunächst sei jedoch ein kurzer Blick auf die Verhältnisse während der vorangehenden Periode der römischen Kaiserzeit (d. h. vom frühen 1. bis zum mittleren 3 . Jahrhundert n. Chr.) geworfen. Antike Städte stellten ,Schrifträume‘ par exellence dar. Diese umfassten nicht nur in dauerhafte Materialien gefasste Texte, also Inschriften im engeren Sinne, sondern auch Schriftzeugnisse auf Holztafeln, auf Wänden aufgemalte oder eingeritzte Texte (Dipinti und Graffiti) etc. Eine besondere Verdichtung von solchen beschrifteten Artefakten lässt sich auf den zentralen öffentlichen Plätzen der römischen Städte, den Fora, ausmachen. Hierbei spielten insbesondere die sog. ,civic inscriptions‘ eine wichtige Rolle, also Ehreninschriften für Kaiser und herausragende Persönlichkeiten auf den Basen von statuarischen Denkmälern sowie Bauinschriften an den öffentlichen Gebäuden, die den Platz rahmten. Im 1. und 2. Jahrhundert hatten sich solche Plätze mit zahlreichen (epigraphischen) Monumenten gefüllt, und zwar in einem solchen Maße, dass man mancherorts Teile dieses Bestandes abräumen musste, um einen ungehinderten Durchgang zu gewährleisten.

Der Höhepunkt der epigraphischen Produktion, die zudem eine große Menge von Weihe- und vor allem Grabinschriften umfasste, wurde in den einzelnen Regionen des (westlichen) Imperium Romanum zu unterschiedlichen Zeitpunkten während des 2. oder früheren 3. Jahrhunderts erreicht. Bemerkenswert ist auf jeden Fall, in welchem quantitativen und qualitativen Umfang im Römischen Reich textuelle Botschaften in ein dauerhaftes Material (Stein, teilweise auch Bronze) gefasst und einer 
mehr oder minder breiten Öffentlichkeit präsentiert wurden. Solche ,monumentalen“ Inschriften hatten (mindestens) eine zweifache Funktion: Sie dienten einerseits als Medien der gesellschaftlichen Kommunikation im öffentlichen und halböffentlichen Raum der Städte, zu dem oftmals auch die Heiligtums- und Grabbezirke zu zählen sind; und sie waren andererseits - gerade durch ihre nicht selten explizit im Text der Inschriften selbst hervorgehobene Dauerhaftigkeit - ,Speicher' der Erinnerung (memoria) an einzelne Persönlichkeiten und Aktivitäten. Diese kommemorative Funktion wurde dadurch verstärkt, dass einzelne epigraphische Monumente nicht selten über Jahrhunderte hinweg im Stadtraum präsent waren. Die im kulturgeschichtlichen Vergleich durchaus eigentümliche Form der Kommunikation und Kommemoration durch eine massenhafte Herstellung und Präsentation von auf Dauer ausgerichteten, beschrifteten Artefakten hat der amerikanische Althistoriker Ramsay MacMullen in einem wegweisenden Aufsatz als den spezifisch antiken ,epigraphic habit‘ bezeichnet.

Mit Blick auf die Spätantike lassen sich nun deutliche Veränderungen in dem solchermaßen definierten ,epigraphic habit‘ ausmachen. Bevor diese näher in Augenschein genommen werden, soll kurz auf die zeitlichen Begrenzungen der Epoche ,Spätantike‘ eingegangen werden. Bei der Betrachtung der spätantiken Stadtentwicklung lässt sich ein Modell entwickeln, das von einer chronologischen Zweiteilung ausgeht: Auf eine noch stark von antiken Traditionen geprägte, late antique city des späten 3. bis frühen 5. Jahrhunderts folgte als weitere Phase eine ,later late antique city im mittleren/späteren 5. sowie im 6. Jahrhundert, die von markanten Transformationsprozessen gekennzeichnet gewesen ist. Diese Einteilung lässt sich, wie gleich zu zeigen sein wird, durchaus auf die Ausprägung der spätantiken Inschriftenkultur(en) in Italien übertragen. Ein erster deutlicher Wandel ist für die Zeit nach der Mitte des 3. Jahrhunderts auszumachen. Dieser manifestierte sich zunächst einmal in einem starken quantitativen Rückgang der epigraphischen Produktion, der augenscheinlich fast alle Inschriftengattungen betraf. Im Falle der - zahlenmäßig am stärksten vertretenen - Weihe- und Grabinschriften ist dies aufgrund oftmals nur sehr vager (und bisweilen eher subjektiver) Datierungskriterien allerdings nicht ganz eindeutig aufzuzeigen. Sehr viel klarer nachzuvollziehen ist die verminderte Präsenz neu gefertigter ,civic inscriptions'. Ihre Zahl ging ab dem mittleren 3. Jahrhundert zurück, allerdings keineswegs auf Null. So wurden Ehreninschriften für Kaiser auf Statuenbasen in der tetrarchisch-konstantinischen Epoche noch recht häufig gesetzt. Auch Bau- bzw. Restaurierungsinschriften wurden in dieser Zeit an öffentlichen Gebäuden angebracht. Am stärksten betroffen von dem Rückgang waren unzweifelhaft die Ehreninschriften für Angehörige der lokalen städtischen Eliten. Diese verschwanden mancherorts bereits im Laufe des 3. Jahrhunderts mehr oder minder vollständig. Allerdings zeigen sich hierbei nun sehr viel deutlicher als während der Kaiserzeit regionale und subregionale Unterschiede: In ,konservativeren' Regionen hielt sich nämlich die Sitte, auch Personen, die nicht Angehörige des Kaiserhauses oder der Reichsaristokratie waren, mit einer Inschrift und einer zugehörigen Statue zu ehren, 
deutlich länger als anderswo. Solche Unterschiede zeigen sich auch innerhalb Italiens: In Norditalien fehlen spätantike Ehreninschriften für Mitglieder der munizipalen Eliten fast völlig, in Mittel- und Süditalien sind sie hingegen noch in einiger Zahl vorhanden, vor allem aus der zweiten Hälfte des 4. Jahrhunderts - in einer Zeit, zu der wiederum kaum noch Ehrenmonumente für Kaiser auf den zentralen Plätzen der Städte errichtet wurden.

$\mathrm{Zu}$ diesen quantitativen Veränderungen kamen qualitative Wandlungsprozesse. Diese betrafen einerseits das Layout der Inschriften: Die stark standardisierten Schriftbilder der Kaiserzeit, welche durch große, rastermäßig angeordnete und tief eingeschnittene Buchstaben charakterisiert waren, wurden oftmals ersetzt durch sehr viel unregelmäßiger und - zumindest für moderne Augen - ,nachlässiger` ausgeführte Schriftgestaltungen. Ob hierfür ein allgemeiner Verfall der handwerklichen Fähigkeiten während der Spätantike verantwortlich zu machen ist, dürfte angesichts von hochwertigen Produkten der Steinmetzkunst auf anderen Sektoren (etwa bei Sarkophagen) zu bezweifeln sein. Gegen eine solche Annahme spricht zudem, dass bisweilen durchaus noch Inschriften hergestellt wurden, bei deren Gestaltung man bewusst an frühere Traditionen anzuknüpfen versuchte. In einzelnen Fällen kam es sogar zu kunstvollen epigraphischen Neuschöpfungen wie denjenigen, die der Bischof von Rom, Damasus, und sein Inschriftenlayouter Philocalus kreierten und dazu nutzten, um die Märtyrerverehrung in den urbanen und insbesondere den suburbanen Raum der Stadt Rom gleichsam einzuschreiben. Diese spezifische Form der Inschriftengestaltung fand zwar nur relativ wenige direkte Nachahmer; sie weist aber auf jeden Fall darauf hin, dass es in der Spätantike eine große Bandbreite in den äußeren Erscheinungsformen von Inschriften gab, so dass insgesamt eine erheblich größere Heterogenität vorherrschte als in der Kaiserzeit. Hiermit verbunden waren vermutlich Veränderungen der Sehgewohnheiten auf Seiten der Rezipienten solcher beschrifteten Artefakte.

Eine weitere wichtige Entwicklung betraf den Umgang mit älteren epigraphischen Monumenten. Ab der Mitte des 3. Jahrhunderts nahm die Wiederverwendung früherer Inschriftensteine als Spolien dramatisch zu. Dies fügte sich in einen allgemeinen Trend ein, denn die massenhafte Verwendung von Spolien sowie die Umbzw. Neunutzung älterer Denkmäler waren charakteristische Merkmale des Städtelebens der Spätantike. In Bezug auf die Inschriften lassen sich hierbei verschiedene Praktiken ausmachen: Zum einen wurden beschriftete Steine pragmatisch als Baumaterial genutzt, nicht selten in Fundamenten, wo sie dem Blick der Betrachter entzogen waren. Bisweilen wurden sie auch sichtbar in neuen Baukontexten platziert. In unserem Kontext besonders interessant ist aber die Wiederverwendung älterer Inschriftenträger mit dem Zweck, diese erneut zu beschreiben. Dies trifft vor allem auf Statuenbasen $\mathrm{zu}$, die in einigen Fällen so oft gedreht und neu beschrieben wurden, bis alle vier Seiten mit einer Inschrift versehen waren. Bisweilen wurden ältere Basen augenscheinlich bewusst wegen des Inhalts ihrer Inschrift(en) ausgesucht und somit gezielt wiederverwendet. 
Diese Entwicklungen lassen sich anhand des erhaltenen Bestandes an Inschriften gut beschreiben; und die generellen Trends haben sich durch die Neufunde der letzten Jahre nicht verändert. Schwieriger ist es, die Wandlungen in der epigraphischen Praxis, die immerhin zentrale Sektoren derselben betrafen, hinreichend zu erklären. Der markante quantitative Rückgang bei der Neuproduktion von Inschriften kann kaum alleine mit einer dramatisch veränderten ökonomischen Situation im Imperium Romanum begründet werden. Gegen eine solche These spricht vor allem die Tatsache, dass es Regionen wie etwa Aquitanien gab, die während der Spätantike unzweifelhaft ökonomisch prosperierten und von einer wohlhabenden Elite, die tief in der klassischen Bildung verwurzelt war, dominiert wurden, wo jedoch dennoch in dieser Epoche kaum noch neue Inschriften errichtet wurden. Hier scheinen Inschriften ihre zentrale Rolle als Medien der sozialen Kommunikation und Kommemoration zunehmend an andere Bedeutungsträger verloren zu haben. Das verweist wiederum auf einen weiteren wichtigen Faktor: Trotz der ausgeprägten politisch-militärischen Krise während des 3. Jahrhunderts ist nicht nachzuweisen, dass es zu einem radikalen gesellschaftlichen Umbruch gekommen wäre, der den veränderten Umgang mit bzw. den Verzicht auf Inschriften im 4. Jahrhundert erklären könnte. Vielmehr muss man wohl davon ausgehen, dass anstelle der epigraphischen Monumente andere Formen der gesellschaftlichen Hervorhebung und Repräsentation an Bedeutung gewannen, darunter solche von ephemerer Natur. Hierzu zählten performative Inszenierungen wie etwa Akklamationen, welche die Volksmenge in einer Spielstätte oder in den Straßen einer Stadt auf einen Wohltäter oder verdienten Mitbürger ausbrachte. Wir erfahren hiervon gelegentlich durch literarische Berichte oder Darstellungen auf den Mosaikfußböden reicher Wohnhäuser. Interessanterweise wurden solche Akklamationen teilweise selbst wiederum inschriftlich aufgezeichnet, so durch Graffiti, die man am Ort des Ereignisses in steinerne Untergründe ritzte.

Weiterhin gilt es zu fragen, wie sich die Räume, die bislang bevorzugt zur Aufstellung bzw. Anbringung von Inschriften gedient hatten, aufgrund der gewandelten epigraphischen Praxis während der Spätantike entwickelt haben. Hierbei sind mit Blick auf das 4. Jahrhundert sowohl Kontinuitäten wie auch Veränderungen zu beobachten. Die Fora blieben in Italien zunächst wichtige ,Schrifträume‘, auch wenn sie nun immer seltener zur Aufstellung neuer epigraphischer Monumente genutzt wurden. Vielmehr kam es verschiedentlich zur Umsetzung älterer Denkmäler auf die Plätze, die im Zuge solcher Maßnahmen mit neuen Beschriftungen versehen wurden. Ein Beispiel hierfür stellt das Forum von Aquileia dar, das im Laufe des 4. Jahrhunderts in eine Art ,Schauraum‘ der Stadtgeschichte verwandelt wurde, bei dessen Gestaltung Inschriften nach wie vor eine wichtige Rolle spielten. Auffällig ist dabei allerdings, dass epigraphisch-statuarische Monumente zu Ehren der regierenden Kaiser auf dem Forum von Aquileia (und auch in anderen Städten) nur noch bis zur Mitte des 4. Jahrhunderts errichtet wurden, obwohl doch die Verehrung des Herrschers weiterhin wichtig war. An diesem Punkt ist auf eine bedeutsame topologische Verlagerung zu verweisen, die in der Forschung bislang noch nicht genügend Beachtung 
gefunden hat: Während die Zahl der Ehreninschriften für Kaiser auf Statuenbasen, welche im öffentlichen Raum der Städte zur Aufstellung kamen, im Laufe des späteren 4. Jahrhunderts stark zurückging, nahm gleichzeitig zumindest in Norditalien die Herstellung von Meilensteinen noch einmal zu. Meilensteine, die entlang der Fernstraßen im Territorium der Städte errichtet wurden, stellten somit eine der wenigen Inschriftengattungen dar, deren Quantität zu Beginn der Spätantike nicht rückläufig war, sondern im Gegenteil deutlich anwuchs. Gleichzeitig hatte sich der Charakter der Meilensteine verändert, wie wir vor allem an dem Formular der auf ihnen angebrachten Inschriften ablesen können. Sie dienten nun nicht mehr vorrangig zur Erinnerung an Straßenbaumaßnahmen oder zur Anzeige der Entfernung vom nächsten Hauptort, sondern als Monumente zur Ehrung des Kaisers entlang der Fernstraßen. Auch Meilensteine stellten somit eine eminent,öffentliche‘ Inschriftengattung dar und sind daher $\mathrm{zu}$ den ,civic inscriptions' $\mathrm{zu}$ rechnen. Das erkennt man auch an ihrer Platzierung: Diese erfolgte zwar in der Regel nicht im Stadtraum, aber oftmals an bewusst gewählten, markanten Punkten an den Straßen (etwa Raststationen und Brücken), wo nicht selten ganze Gruppen von Meilensteinen zur Aufstellung kamen, die dadurch eine eigene Form von Monumentalität entwickelten, auch wenn ihre Inschriften auf den gerundeten Säulen nicht einfach zu lesen waren - auf Letzteres kam es dabei offenbar nicht primär an.

In dieser Phase hat augenscheinlich das Christentum noch keinen dominanten Einfluss auf die epigraphische Praxis gehabt. Zwar gewann die Christianisierung des Imperium Romanum seit der ,konstantinischen Wende“ im Laufe des 4. Jahrhunderts zunehmend an Dynamik, aber im öffentlichen Raum der Städte machte sich dies zunächst kaum bemerkbar, zumal die Errichtung von Kirchenbauten vielerorts nicht vor dem späten 4. Jahrhundert einsetzte. Die Kirche besetzte die zentralen Plätze nicht mit eigenen epigraphischen Monumenten; vielmehr äußerten sich einige Kirchenväter negativ gegenüber den traditionellen Foki des städtischen Lebens. So kennen wir aus der Spätantike praktisch keine öffentlichen Ehrungen für Bischöfe durch Inschriften und Statuen. Da aber gleichzeitig auch dezidiert ,pagane' Statements in den öffentlich präsentierten Inschriften während des 4. Jahrhunderts nur noch sehr selten vorkamen, hat man von einem religiös ,neutralen' Raum gesprochen, welcher weite Bereiche der Städte während der früheren Spätantike geprägt habe. Die bereits angesprochene Umsetzung von Götterstatuen auf die Fora steht hierzu nicht im Widerspruch, da die in diesem Kontext in der Regel vorgenommenen Neubeschriftungen der Basen nicht mehr auf den religiösen Gehalt der Standbilder abhoben, sondern auf die hierdurch bewirkte Verbesserung des ,Schmuckes“ des Platzes. Jedenfalls handelte es sich hierbei nicht mehr um traditionelle Weiheinschriften. Im funerären Kontext schließlich machte sich die Ausprägung distinktiv christlicher Formeln und Symbole außerhalb von Rom häufig ebenfalls erst im mittleren bzw. späteren 4. Jahrhundert bemerkbar, auch wenn auf breitere Überlappungszonen - sowohl in chronologischer wie auch in räumlicher Sicht - hinzuweisen ist. 
Während somit die erste Phase der spätantiken Inschriftenkultur trotz des starken Rückgangs der epigraphischen Produktion von klar auszumachenden Kontinuitätslinien zu dem antiken ,epigraphic habit' gekennzeichnet war, lassen sich ab dem früheren 5. Jahrhundert nochmals sehr deutliche Veränderungen beobachten. Am auffälligsten ist dabei sicherlich, dass nunmehr die ,civic inscriptions` (nach der oben angeführten Definition) in Italien fast völlig verschwanden. Besonders eindrücklich ist die Feststellung, dass es aus der Zeit nach 423 n. Chr. kaum noch ,Kaiserinschriften' aus Italien gibt, also Ehreninschriften für den Herrscher sowie Bauinschriften, in denen sein Name verzeichnet wurde. Dies gilt nicht nur für die nur kurzzeitig regierenden Kaiser der zweiten Hälfte des 5. Jahrhunderts, sondern auch schon für Valentinian III., der von 425 bis 455 an der Spitze des weströmischen Reiches stand - für ihn hat sich keine einzige Ehreninschrift erhalten, nicht einmal in Rom! Ebenfalls bemerkenswert ist, dass selbst die Errichtung von Meilensteinen, die während des 4. Jahrhunderts, wie gesehen, noch zugenommen hatte, zu Beginn des 5. Jahrhunderts vollkommen abbrach; diese Praxis wurde danach nie wieder aufgenommen. Diese Trends kehrten sich im Übrigen auch während der Regentschaft des Ostgotenkönigs Theoderich (493-526) in Italien nicht mehr um.

Eine weitere wichtige Veränderung in dieser Phase betraf die topologische Verortung der Inschriften. Der frei zugängliche, öffentliche Raum der Städte mit den zentralen Platzanlagen, der in der Kaiserzeit und teilweise auch noch zu Beginn der Spätantike der wichtigste Ort für die Platzierung von Inschriften, welche für ein breiteres Publikum konzipiert waren, gewesen war, verlor parallel zum Verschwinden der ,civic inscriptions' stark an Bedeutung. Ein deutliches Indiz hierfür ist, dass in vielen Städten Italiens die Fora im Laufe des 5. Jahrhunderts aufgelassen oder überbaut wurden. Gleichzeitig nahm nun die Zahl neu errichteter, größerer Gemeindekirchen zu. Hierdurch standen neue ,Inschriftenräume‘ zur Verfügung, die tatsächlich intensiv genutzt wurden. Dort kamen auch neue Materialien zum Einsatz, die sich besonders für die Ausgestaltung von Innenräumen eigneten. Hierbei spielten in bestimmten Regionen Italiens insbesondere in Mosaik ausgeführte Texte des 5. und 6. Jahrhunderts auf den Fußböden und an den Wänden von Kirchenbauten eine wichtige Rolle. Diese Mosaiktexte konnten einerseits in ausführlicher Form und an einem prominenten Platz, etwa in der Apsis oder am ,Triumphbogen', als ,Hauptbauinschrift“ die Errichtung einer Kirche kommemorieren, wobei hierbei häufig dem Bischof eine bedeutende Position zugewiesen wurde. Anderseits wurden auf den Mosaikfußböden der Kirchen in Nordost-Italien zahlreiche kleine Schriftfelder eingebracht, in denen in knapp gehaltenen Texten die Zuwendungen von Gemeindemitgliedern - und zwar sowohl von Mitgliedern der sozialen Elite als auch von ,kleinen Leuten' - für den Ausbau der Kirchen festgehalten wurden. Dabei kam sicherlich teilweise eine neue christliche Wertewelt zum Tragen, bei der es etwa für die Stifter um die Erlangung des Seelenheiles vor Gott ging oder auch darum, durch die schriftliche Fixierung ihres Namens die Einbeziehung in das Gebetsgedenken zu sichern. Mosaikinschriften, die solche Gedanken explizit abriefen, indem sie beispielsweise auf die Nennung von 
Namen bewusst verzichteten und dadurch anonym blieben, sind durchaus vorhanden, aber nur in recht geringer Zahl. Die Masse der Schriftzeugnisse auf den Mosaikfußböden der Kirchen stellt hingegen den Namen des Stifters und nicht selten auch seinen sozialen Rang sowie die Höhe seiner Zuwendungen deutlich heraus, was in Verbindung mit der votum-Formel durchaus als eine Kontinuitätslinie zu früheren euergetischen Inschriften angesehen werden kann. Zudem lässt sich an der Platzierung und Gestaltung (etwa durch farbig gefasste Buchstaben oder Unterstrichlinien) der Mosaikinschriften erkennen, dass diese die Aufmerksamkeit der Betrachter erregen sollten. Sie hatten somit durchaus (auch) eine Repräsentationsfunktion, welche die Leistungen von Einzelpersonen vor der Gemeinschaft hervorhob. Mit solchen Schriftartefakten wurde also immer noch ein größeres städtisches Publikum angesprochen.

Man kann daher postulieren, dass die Kirchenbauten als ,Inschriftenräume‘ in gewisser Weise die Rolle der zu dieser Zeit zunehmend außer Benutzung geratenden großen Platzanlagen in den Städten Italiens übernommen haben. Dabei ist allerdings auf einen gewichtigen Unterschied hinzuweisen: Selbst die oben angeführten ,Hauptbauinschriften' der Kirchen waren zumeist im Innenraum der Gebäude (etwa an der Innenwand über dem Hauptportal) angebracht und eben nicht an deren - zumeist eher schmucklos gestalteten - Außenfassaden, was hingegen bei den Bauinschriften an den öffentlichen Gebäuden in den römischen Städten die Regel gewesen war. Man kann hier also von einer ,Interiorisierung، der epigraphischen Kommunikation sprechen.

Dazu passt der Befund, dass der zweite in dieser Phase verbliebene, bedeutende ,Inschriftenraum' durch funeräre Kontexte gebildet wurde. Diese konnten durchaus auf ein breiteres Publikum ausgerichtet sein, etwa in einigen vorstädtischen Nekropolen im Umkreis der Ausfallstraßen, in denen beschriftete Sarkophage oberirdisch zur Aufstellung kamen. Vielfach war aber die Wahrnehmung von spätantiken Grabinschriften, die durch den Einsatz von Ritzzeichnungen durchaus anspruchsvoll gestaltet sein konnten, durch eine ,restringierte Präsenz' gekennzeichnet, also durch eine gewisse Abgeschiedenheit, die nur zu bestimmten Anlässen, etwa anlässlich von Toten-Gedenktagen, von kleineren Gruppen von Familienangehörigen aufgesucht wurde. Daneben muss auch dem Akt der Bestattung selbst einige Bedeutung zugekommen sein, denn hier dürfte das beschriftete Grabmal einer größeren Zahl von Betrachtern vor Augen gestanden haben - was vielleicht auch erklären mag, warum manche Sarkophage anschließend in die Erde versenkt wurden, wo ihre Inschriften fortan überhaupt keinem Betrachter mehr zugänglich waren. Nicht wenige dieser Grabinschriften wiesen mehr oder minder dezidiert christliche Formeln und Symbole auf, was kaum verwundert, war doch die römische Gesellschaft zumindest nominell ab dem ausgehenden 4. Jahrhundert weitgehend christianisiert. Ein solcher christlicher ,epigraphic habit‘ am Grab hatte sich im Laufe des 4. Jahrhunderts herausgebildet. Es gab daneben aber nicht wenige Grabinschriften, denen es an einer eindeutigen religiösen Kennzeichnung mangelte. Es ist daher zu betonen, dass man - gerade mit Blick auf die gesamte epigraphische Produktion der Spätantike - diese Inschriften- 
kultur nicht einseitig als eine ,christliche“ charakterisieren, sondern besser allgemein von ,spätantiker Epigraphik‘ sprechen sollte.

Zusammenfassend kann man festhalten, dass auch während des 5. und 6. Jahrhunderts, also in der Endphase der Spätantike, noch recht zahlreiche Inschriften sowohl in Stein wie auch als Mosaik gefertigt wurden - jedenfalls deutlich mehr als in der nachfolgenden Periode des beginnenden Frühmittelalters im 7. und 8. Jahrhundert. Diese Inschriften waren nun aber nur noch auf wenige Aufstellungsorte konzentriert, insbesondere Kirchenräume und Grabkontexte, während der öffentliche Raum der Städte, der durch die gleichzeitigen Veränderungen der Stadtbilder ohnehin zunehmend an Bedeutung einbüßte bzw. gänzlich aufgelassen wurde, hierfür kaum noch genutzt wurde. Man kann sich angesichts dieser Entwicklungen fragen, inwiefern die Inschriftenkultur dieser Periode überhaupt noch ,(spät)antik‘ geprägt war. In der Tat ist der Übergangscharakter dieser Epoche nicht zu übersehen, denn nicht wenige der hier herausgestellten Charakteristika treffen in ähnlicher Form auch auf die frühmittelalterliche Periode zu. Dennoch scheinen an einigen Punkten die Verbindungslinien zu der Inschriftenkultur der vorangegangenen Zeit so stark gewesen zu sein, dass es durchaus gerechtfertigt erscheint, auch die epigraphische Praxis des 5. und 6. Jahrhunderts im Kern noch als ,spätantik` zu bezeichnen.

\section{Ergebnisse: Praxeologisch-topologische Zugänge}

Die Zusammenschau der in diesem Band versammelten zehn Beiträge lässt jenseits der hier gewählten Anordnung weitere Schwerpunktbildungen erkennen: Besonders intensiv widmen sich viele Beiträge Fragen der ,Topologie‘, also der Beschreibung der räumlichen Anordnung von schrifttragenden Artefakten und den Bedingungen ihrer Platzierung im Raum. Dies kann nicht überraschen, standen doch bei der 2016 in Rom durchgeführten Tagung die ,ausgestellten Schriftzeugnisse“ („scritture esposte“) im Zentrum der Aufmerksamkeit. Ausstellungs- und Anbringungsort von Inschriften nehmen insbesondere in den Beiträgen von Nicoletta Giovè, Vincent Debiais, Katharina Bolle und Henrike Haug eine wichtige Stellung ein. Das Verhältnis zwischen städtischer Infrastruktur und Inschrift lässt sich vor allem an neuralgischen Knotenpunkten des urbanen Raumes wie Stadttoren oder Straßenkreuzungen aufzeigen. Inschriften waren dort oftmals exponiert angebracht und damit auf Sichtbarkeit angelegt; sie verstärkten in aller Regel Kommunikationssituationen, die bereits existierten. Die kombinierte Untersuchung von Inschriftenpräsenz und -topographie erweist sich als ein wertvolles heuristisches Instrument, denn an dem untersuchten epigraphischen Material kann aufgezeigt werden, wie Räume in den Städten des mittelalterlichen Italien durch prononciert platzierte Inschriften Veränderungen erfuhren und ihrerseits die Kommunikationssituation zwischen Artefakt und Betrachter prägten. Die gewählten Fallbeispiele bestätigen zudem die in der Einleitung formu- 
lierte Vermutung, dass in italienischen Städten des 11.-13. Jahrhunderts die antike Tradition verstärkt aufgegriffen wurde, an neuralgischen Orten Inschriften politischen Inhalts bzw. ,civic inscriptions' anzubringen, welche die Stadt, die Kommune und/oder führende Familien feierten.

Die meisten Autoren fokussieren die ,Mikrotopographie‘ des jeweiligen beschrifteten Artefakts, also seine Positionierung an einem bestimmten Platz innerhalb der Stadt, etwa auf einer Brücke o. ä. Wie Vincent Debiais zeigen kann, wurde diese Anbringungssituation in steinernen Urkunden nicht nur dinglich durch ihren Ausstellungsort, sondern ausdrücklich auch im Text der Inschrift selbst hervorgehoben („dieser Turm“, „diese Mauer“, „dieses Tor“). Ähnlich demonstrative Verweise können Rebecca Müller für Genua, Nicoletta Giovè für Viterbo, Flavia De Rubeis für Padua, Wilfried Keil für Mailand und Katharina Bolle für Rom anführen. Andere Beiträge hingegen - allen voran die Aufsätze von Arnold Esch und Marialuisa Bottazzi - fokussieren stärker die ,Makrotopographie‘ räumlicher Gefüge, indem sie dezidiert das gesamte Stadtbild in ihren Überlegungen einbeziehen.

Bei aller gebotenen Vorsicht lassen sich im Vergleich durchaus ,epigraphic habits in den einzelnen Orten herausarbeiten, die als Ausdruck eines städtischen oder gar kommunalen Selbstverständnisses zu interpretieren sind. Vorsichtig werden solche wiedererkennbaren inschriftlichen Merkmale von Rebecca Müller für Genua vorgeschlagen, offensiver hingegen von Henrike Haug postuliert. Der Beitrag von Arnold Esch propagiert für Pisa eine ähnliche Sonderstellung, die Katharina Bolle und Marialusia Bottazzi ihrerseits dezidiert für Rom einfordern. Einen ,epigraphic habit‘ ganz anderer Art konturiert Vincent Debiais, indem er nach den Spezifika einer steinernen Inschrift gegenüber einer Urkunde fragt. Mit Recht weist er darauf hin, dass die „chartes lapidaires“ keineswegs die schlichte Versteinerung eines Schriftstücks darstellten, sondern eigene Charakteristika aufwiesen.

Der praktische Umgang mit schrifttragenden Artefakten in mittelalterlichen Städten ist bekanntlich in der Regel schwer im Detail zu rekonstruieren. Trotz dieser Herausforderung haben die meisten Autoren bzw. Autorinnen den Versuch unternommen, eine praxeologische Perspektive einzunehmen. Mittels einschlägiger Metatexte, die vom Umgang mit schrifttragenden Artefakten berichten, aber auch durch eingehende Untersuchungen epigraphischer Interdependenzen leisten sie damit einen wertvollen Beitrag zur Praxeographie von Inschriften im urbanen Gefüge des Mittelalters. Dies gilt auch für diejenigen Aufsätze, die sich mit der Wiederverwendung älterer Inschriften beschäftigen. Die Möglichkeiten eines solchen gezielten Umgangs mit bereits bestehenden Artefakten im Mittelalter wiesen bekanntlich ein beträchtliches Spektrum auf, wie auch die Beiträge dieses Sammelbandes eindrücklich demonstrieren. Die Verwendung eines älteren Objekts im Sinne einer Trophäe wird gleich mehrfach ausführlich behandelt, vor allem am Falle Genuas (Rebecca Müller, Nicoletta Giovè, Wilfried Keil), ebenso die Translozierung älterer Inschriften in neu errichtete Gebäude (etwa im Falle Pisas: Henrike Haug), aber auch die gezielte Beschädigung oder Zerstörung schrifttragender Artefakte (Wilfried Keil). 
Eine praxeologische Untersuchung städtischer Epigraphik fragt notwendigerweise auch danach, welche städtischen (Teil)Öffentlichkeiten die epigraphischen Erzeugnisse überhaupt zu Gesicht bekamen, welche Gruppen die Texte verstehen konnten und wie zeitgenössische Beobachter auf Inschriften im öffentlichen Raum reagierten. Mit Recht warnt Arnold Esch in seinem Beitrag vor einer Überbetonung des gelehrten Lektors, und in der Tat mahnen die von ihm ins Auge gefassten kleinen und ländlichen Kirchen in dieser Hinsicht zur Vorsicht. Dezidiert hat diesen Aspekt ferner Katharina Bolle aufgegriffen, indem sie Belege für die Lesbarkeit und das tatsächliche Lesen von antiken Inschriften im mittelalterlichen Rom vorlegt und damit Wege für eine zukünftige Erforschung mittelalterlicher Lesegewohnheiten und epigraphischen Wissens aufzeigt. Auch Lukas Clemens und Erik Beck liefern aufschlussreiche Hinweise auf die gezielte Funktionalisierung lesbarer, gelesener und in unterschiedlichem Ausmaß verstandener Inschriften. Henrike Haug wiederum differenziert anhand Pisaner und Genueser Beispiele unterschiedliche Rezipientenkreise epigraphischer Kommunikation - die Stadtbewohner, die Stadtbesucher und die Beobachter von Inschriften in entfernten Städten (hier Jerusalem). Vincent Debiais schließlich weist in Steinurkunden die ausdrückliche Aufforderung zum Lesen selbst schwer zu entziffernder Texte nach. Die in den Beiträgen herausgearbeiteten, unterschiedlichen Formen des praktischen Umgangs mit epigraphischen Monumenten belegen eindrücklich, dass mittelalterliche Inschriften in urbanen Kontexten in der Tat als ein „hyperkommunikatives“ Medium (Nicoletta Giovè) fungierten.

Die Aufsätze konkretisieren und vertiefen nicht nur anerkanntes Wissen, sondern stellen in ihrer Gesamtschau auch einige Axiome auf den Prüfstand - darunter auch eine Grundannahme der diesem Band zugrunde liegenden Tagung. Denn sie geben zur Frage Anlass, ob überhaupt von einer „kommunalen Inschriftenkultur“ die Rede sein könne: War die Kommune als Verfasstheit der städtischen Eliten wirklich ein so entscheidender Faktor, dass sie einen eigenen epigraphischen Habitus generierte und daher namensgebend für (politische) Inschriften im öffentlichen Raum der Städte sein sollte? Flavia De Rubeis stellt diese Frage mit aller Dringlichkeit. Es mag durchaus bezeichnend sein, dass einige der in diesem Band vorgestellten Fallbeispiele von urbanen Zusammenhängen und dem Feld der politischen Herrschaftsausübung wegführen, so die Dorfkirchen, die Künstler- und Stifterinschriften etc. Diese Phänomene erweitern das Feld und lassen es - wie von De Rubeis vorgeschlagen - angebrachter erscheinen, im Sinne einer Epochenangabe von der „Inschriftenkultur im kommunalen Zeitalter“ anstatt von einer „kommunalen Epigraphik“ zu sprechen. Ist hingegen dezidiert von der Inschriftlichkeit laikaler politischer Eliten in der Stadt die Rede, so mag es geboten erscheinen, in Anlehnung an bereits eingeführte Begriffe - wie „epigrafia civica“ oder „civic epigraphy“ - von einer „politischen Inschriftenkultur“ in der mittelalterlichen Stadt zu sprechen. Schließlich sollte der Terminus der „kommunalen Inschriftenkultur“ den spezifischen und im europäischen Vergleich seltenen Ausprägungen mittelalterlicher Epigraphik vorbehalten sein, die im Sinne der Einlei- 
tung zu diesem Band von kommunal verfassten städtischen Einrichtungen bzw. deren Führungspersonen im öffentlichen, nicht-kirchlichen Raum ausgestellt wurden.

Eine solche dreifache begriffliche Unterscheidung würde es auch erleichtern, analytisch zwischen familiärer und korporativer Epigraphik zu unterscheiden, wie dies Flavia De Rubeis prominent betreibt, Rebecca Müller im Falle Genuas vorführt und auch Nicoletta Giovè aufgreift. Auch wenn De Rubeis letztlich der Funktion einer Inschrift den epistemischen Vorrang über deren Auftraggeber einräumt, so zeigen die in diesem Band versammelten Beiträge dennoch eindrücklich, wie lohnend eine Untersuchung der Inschriftlichkeit städtischer korporativer Führungsgruppen sein kann. Die in den Aufsätzen immer wieder aufscheinende Verknüpfung von kollektiver historischer Erinnerung, der Hervorhebung individueller städtischer Führungspersönlichkeiten oder Amtsträger und der Anbringung von Inschriften an Orten verdichteten Verkehrs belegt die sprunghaft verstärkte Nutzung politischer Epigraphik im kommunalen Zeitalter. Inschriften dienten in diesen Fällen als Ausdruck von Macht - nicht nur über eine Stadt oder eine Gemeinschaft, sondern auch über den öffentlichen Raum in der Stadt, wie die von Nicoletta Giovè vorgestellten Brunnen besonders prominent illustrieren. Vereinzelt lässt sich hierbei ein Ringen um die Symboltopographie feststellen, denn der Name eines Bauherrn oder anderer Auftraggeber in einer öffentlich präsentierten Inschrift hielt nicht zuletzt fest, dass eben er und niemand anderes die Macht besaß, eine solche zu setzen. In der Tat zeigen diese und andere Beispiele, dass die spezifische Positionierung eines auf Dauer ausgerichteten epigraphischen Monuments immer auch Ausdruck von Herrschaft über den ,beschreibbaren' Raum war - und noch immer ist.

Eine weitere analytische Scheidung, zu der die hier versammelten Beiträge einladen, ist diejenige zwischen ,Sichtbarkeit' und ,Lesbarkeit‘. Inschriften tatsächlich (vollständig) inhaltlich durchdringen und verstehen zu können, war im kommunalen Zeitalter offenbar viel seltener nötig oder intendiert, als es oftmals in der Forschung angenommen wird. Anbringungshöhe und Lesbarkeit eines beschrifteten Artefaktes werden aus dieser Perspektive zu aufschlussreichen Faktoren der wissenschaftlichen Interpretation. Offenbar war allein die Präsenz einer Inschrift oftmals bereits Botschaft genug. Die - zumindest potentiell zumeist gegebene - Lesbarkeit einer Inschrift funktionierte aus dieser Perspektive eher als ,aide mèmoire‘, welche verbreitetes, mündlich tradiertes Wissen bei Bedarf offiziell und verbindlich abrufbar machte, denn als alltäglich zur Kenntnis genommene Setzung dieses Wissens.

Wenn man von dieser Prämisse ausgehend das Augenmerk stärker auf die demonstrative Sichtbarkeit einer Inschrift als auf deren Lesbarkeit legt, so erlangt das Layout folgerichtig einen erhöhten epistemischen Wert. Vincent Debiais widmet sich diesem Phänomen besonders intensiv. Durch die visuelle Betonung des Wortes juramus konnten beispielsweise Steinurkunden die performative Inszenierung von Rechtshandlungen monumental in Erinnerung rufen und prospektiv für die Zukunft vorschreiben. Auch andere Beiträge gehen dezidiert auf die graphische Gestaltung mittelalterlicher Inschriften ein: Katharina Bolle zum Beispiel konstatiert ein qualita- 
tives Gefälle im Layout und in der technischen Ausführung zwischen kirchlichen und kommunalen Inschriften im Rom des 12. Jahrhunderts; Nicoletta Giovè hebt auf die graphische Qualität der prachtvollen Beschriftungen italienischer Brunnen ab, und Marialuisa Bottazzi betont die Bedeutung des Papstes Damasus für die Gestaltung römischer Inschriften seit dem 4. Jahrhundert.

In anderen Fällen konnte Schrift, auch ohne inhaltlich verstanden zu werden, dadurch eine zusätzliche ,Aura' gewinnen, dass sie als besonders alt und damit als ehrwürdig empfunden oder stilisiert wurde. Die italienischen Kommunen des 11.-13. Jahrhunderts, aber auch andere städtische Gemeinschaften griffen auf antike epigraphische Formen und Materialien zurück. Diese Wertschätzung setzte ein Gespür für die Historizität von Graphie voraus, das verschiedentlich - etwa in den Beiträgen von Arnold Esch, Flavia De Rubeis und Lukas Clemens / Erik Beck - in der Tat identifiziert werden konnte. So weist Esch ein Spielen mit den Zeitindices historischer Artefakte nach, die ausdrücklich in ihrem alten Zustand belassen und nicht nachbearbeitet wurden, weil Inschriftenspolien Alter und Altehrwürdigkeit evozierten und der Beobachter auf diese Weise in Staunen versetzt werden sollte. Dieses ,Grundrauschen“ des Vergangenen war jenseits des konkreten Inhalts des Geschriebenen wesentlich für die Wiederverwendung älterer Inschriften im Mittelalter, wie auch der Beitrag von Marialuisa Bottazzi am Beispiel wiederverwendeter Urnen zeigt. Insofern lässt sich durchaus als ein Ergebnis dieser Untersuchungen festhalten, dass auch in der politischen Epigraphik des kommunalen Zeitalters die Affordanzen schrifttragender Artefakte variabel und sowohl durch das Material als auch durch die Gestaltung der Schrift und deren Inhalt geprägt waren.

\section{Perspektiven: Sechs Felder für zukünftige For- schungen zur mittelalterlichen Inschriftlichkeit}

Wie eingangs und in der Einführung von Marc von der Höh bereits festgehalten, sind die oben skizzierten Fragestellungen eng mit dem Forschungsdesign des SFB 933 „Materiale Textkulturen“ verbunden. Einige methodische Ansätze des Heidelberger Forschungsverbunds wurden weniger stark aufgegriffen, andere hingegen in besonderem Maße vertieft. Welche Perspektiven lassen sich nun abschließend für die mediävistische epigraphische Forschung ableiten? Die Materialien und Formate schrifttragender Artefakte werden insgesamt eher selten in den Blick genommen (wenngleich Nicoletta Giovè zwischen Marmor, Tuffstein, Peperin, Trachyt etc. unterscheidet). Stets handelte es sich bei den hier untersuchten Fallbeispielen um Stein, in den Texte eingemeißelt wurden. Inwiefern ebendiesem Material eine eigene Semantik innewohnte und diesbezüglich unterschiedliche Wertigkeiten des Steins bei der Analyse schriftragender Artefakte in Anschlag zu bringen sind, sind wichtige, wenngleich mangels entsprechender Metatexte schwer zu beantwortende Fragen. Auch wenn 
Vincent Debiais aufschlussreiche Beispiele dafür beibringt, wie im Mittelalter die Nutzung von Stein als Schriftträger ausdrücklich begründet wurde, und Arnold Esch sowie Lukas Clemens und Erik Beck die bewusste Verwendung spezifischer, mitunter von weit her transportierter steinerner Objekte aufzeigen, bleibt hier und bei der Frage nach der Nutzung spezifischer Formate noch Raum für weitere Forschungen.

Auffällig ist ferner, dass neben Palästen und anderen öffentlich wirksamen und in aller Regel zugänglichen Bauten zwar immer wieder Kirchen als Anbringungsorte von Inschriften vorgestellt werden, der Blick sich dabei aber fast ausschließlich auf deren Außenfassaden, nicht aber auf den Innenraum konzentrierte. Der Grund hierfür dürfte in der Erwartung der Tagungsorganisatoren (und Herausgeber dieses Bandes) zu suchen sein, vor allem spezifisch ,kommunale Orte' in den Blick zu nehmen. Damit wurde von den Autoren eine Perspektive eingefordert, die sich in der Praxis als anfechtbar erwiesen hat. Es lässt sich in der Tat mit guten Gründen fragen, ob die Gleichsetzung von ,öffentlichem Raum‘ mit dem für breitere Personengruppen zugänglichen Außenraum einer kritischen Überprüfung standhält. Daher haben einige Autorinnen und Autoren mit Recht vereinzelt Seitenblicke in das Innere von Kirchengebäuden geworfen oder einen Vergleich mit kirchlicher Epigraphik angedeutet (Vincent Debiais, Marialusia Bottazzi, Katharina Bolle u.a.). Zukünftige Forschungen zur politischen Epigraphik der mittelalterlichen Stadt sollten daher weniger stark zwischen Innen- und Außenräumen, zwischen kirchlichen und laikalen Kontexten unterscheiden.

Insgesamt zeigen die hier versammelten Aufsätze deutlich, dass Inschriften in den Städten des kommunalen Zeitalters als ein plurimediales, „hyperkommunikatives“ Mittel fungierten, das vor allem dazu diente, kollektive (auch familiäre) Haltungen zu Vergangenheit, Gegenwart und Zukunft zu stiften. Dies erreichten sie unter anderem durch die Transmission von Gedächtnis, durch ,Propaganda', durch Repräsentation - aber auch durch Vergessen. Inschriften waren - nicht selten gleichzeitig - in verschiedene Kontexte eingebunden und wiesen eine eigene Historizität sowie mitunter komplexe Zeitindices auf. Die Beiträge dieses Bandes laden dazu ein, weiter über solche Zeitebenen, Kontexte und Funktionen nachzudenken. Insbesondere sechs potentiell ertragreiche Forschungsfelder treten nach der Lektüre hervor.

Erstens ist auf dem mit der Tagung und diesem Band eingeschlagenen Weg voranzuschreiten und das Verhältnis der hochmittelalterlichen Inschriftlichkeit zur frühmittelalterlichen und antiken Epigraphik weiter auszuloten. Dieser Beziehung ist nicht allein über die Textinhalte der Inschriften nachzugehen, sondern auch über die in diesem Sammelband angewendeten Fragestellungen nach der Materialität, der Ästhetik, der Paläographie, der Anbringungssituation, der Schriftgestaltung etc. Die Einleitung von Marc von der Höh und die Beiträge von Arnold Esch, Rebecca Müller, Lukas Clemens / Erik Beck, Katharina Bolle und Marialuisa Bottazzi zeigen, wie fruchtbar ein solcher Zugang sein kann. Auch die analytische Unterscheidung zwischen dem durch Abschriften und Sammlungen antiker Inschriften sowie durch 
andere Metatexte tradierten ,Wissen' einerseits und der unmittelbaren ,Anschauung“ älterer Artefakte vor Ort andererseits hat sich bewährt.

Hierdurch wird zugleich die Frage nach der Radikalität der Veränderung von Inschriften-Praktiken im hohen Mittelalter berührt. Ist dabei von einer Transformation im Sinne einer allmählichen Veränderung auszugehen oder eher von einem Umbruch, der sich im hohen Mittelalter zeitgleich zur kommunalen Bewegung und eventuell von dieser beeinflusst vollzog? Ein solcher radikaler Wandel wird in der einschlägigen Forschung unter Hinweis auf paläographische und philologische Befunde postuliert (vgl. etwa den Beitrag von Nicoletta Giovè). Sollten die Entwicklungen hingegen stärker von Kontinuität geprägt gewesen sein, so stellt sich die Frage nach den Orten, Institutionen und Personengruppen, die als Verbindungsglieder zwischen spätantik-frühmittelalterlicher einerseits und hochmittelalterlicher Inschriftlichkeit andererseits gedient haben könnten. Der Beitrag von Marialuisa Bottazzi stellt durch den Hinweis auf die formative Phase unter Bischof Damasus die wichtige Rolle des Papsttums als Stifter von Kontinuität heraus und greift damit eine in der Einleitung von Marc von der Höh aufgeworfene Frage auf. In der Tat dürfte den Pontifices, aber auch der römischen Kurie, die noch immer einer erschöpfenden wissenschaftlichen Aufbereitung aus dieser Perspektive harren, hierbei eine Schlüsselrolle zufallen. ${ }^{1}$

Zweitens zeigen die Beiträge eindrücklich, wie wichtig die Rekonstruktion der konkreten Kontexte urbaner Inschriften ist. Zu diesen Kontexten gehören die Infrastruktur und damit das topographische Umfeld der Artefakte wie auch die Identifizierung und Lokalisierung verlorener Inschriften, die oftmals ganze Inschriftenensembles bildeten. Die Intertextualität und Intermedialität beschrifteter Artefakte im öffentlichen Raum sind wegen der in aller Regel über die Jahrhunderte erfolgten Veränderungen der Topographie und aufgrund des Verlusts einschlägiger Inschriften oftmals nur schwer zu rekonstruieren; dennoch oder gerade deshalb bilden sie eine wichtige Aufgabe einer praxeologisch orientierten Analyse. Herausragende Fälle wie die von Marc von der Höh und Katharina Bolle aufgezeigten Interdependenzen zwischen antiker und mittelalterlicher Inschriftlichkeit auf dem Ponte Cestio zu Rom zeigen das Erkenntnispotenzial dieses Zugangs.

Drittens wecken die in manchen Aufsätzen zutage tretenden Datierungsfragen und die in der Einleitung und andernorts formulierte Erkenntnis, dass mittelalterliche Zeitgenossen durchaus ältere Schriften nachzuahmen vermochten, den Ruf nach einer grundwissenschaftlichen Schärfung des Forschungsfelds. Wenn im hohen und späten Mittelalter prinzipiell die Fähigkeit zur Imitation früherer epigraphischer Schriftcharaktere bestand, so sollte dies unmittelbare Auswirkungen auf unsere Arbeit als Epigraphikerinnen und Epigraphiker haben: Es müssen größere Anstrengungen um das discrimen veri et falsi im Fach und auch um seine Methoden vorgenommen werden.

1 Folgerichtig wird sich das Unterprojekt 3 des Teilprojekts A01 des SFB 933 mit seinem Bearbeiter Dr. Wolf Zöller zukünftig monographisch diesem Forschungsfeld widmen. 
Des Weiteren haben viertens einige Autoren bzw. Autorinnen (u. a. Marialuisa Bottazzi, Arnold Esch) gezeigt, wie lohnenswert ein Blick über das klassische Gebiet der kommunalen Bewegung, also über Nord- und Mittelitalien hinweg, sein kann. Ebenso wie Lukas Clemens und Erik Beck in ihrem Beitrag Vorstellungen von antiker romanitas als ein transalpines Phänomen untersuchen, bietet sich eine geographische Erweiterung unserer Perspektive auf den Süden der Apenninenhalbinsel (wie bei Marialuisa Bottazzi und Flavia De Rubeis bereits angeklungen) und auf andere Regionen im circummediterranen Raum an. Eine solche Erweiterung würde dazu beitragen, auf der analytischen Ebene die politische Inschriftlichkeit des hohen Mittelalters von einer bestimmten Form der Stadtherrschaft - eben der kommunalen Verfassung - zu lösen, und verspricht auch auf der inhaltlichen Seite fruchtbare Erträge.

Fünftens haben die Beiträge unsere Sensibilität für die Weite des Forschungsfelds geschärft. Die Aufsätze vermitteln in ihrer Tiefe und Breite einen Eindruck von der Komplexität dessen, was manchmal etwas undifferenziert als ,Inschriftlichkeit“ bezeichnet werden kann. Tatsächlich verlangt der Begriff nach sachgemäßeren Unterscheidungen, so zum Beispiel nach dem Kriterium der beteiligten Akteure: Dem (stets auch im Plural zu denkenden) Auftragsgeber, Verfasser, Steinmetz, Architekt, Baumeister, Leser usw. Eine solche Differenzierung ist zweifelsohne in der Praxis aufgrund der Quellenlage häufig nur schwer vorzunehmen, doch haben einzelne Beiträge deren Wert vor Augen geführt und Wege $\mathrm{zu}$ ihrer Umsetzung aufgezeigt. Zugleich haben sie das Bewusstsein für die Notwendigkeit geschärft, dezidiert nach den Formen der Zusammenarbeit zwischen diesen Akteuren zu fragen.

Schließlich erlauben es, sechstens, einige der vorgestellten Artefakte, veritable Inschriftenbiographien zu verfolgen. Ob nun die Änderungen von Inschriften (etwa bei der Löwenkopfinschrift in Genua oder den verdeckten Inschriften von Ferrara) oder der Transfer von Inschriften (etwa von Konstantinopel oder von Pisa nach Genua); ob die Überführung antiker Inschriften in das nordalpine Reich nach Konstanz oder Veränderungen des bebauten bzw. allgemein des räumlichen Umfelds: Die in diesem Band behandelten Fallbeispiele zeigen, welche reichen Erkenntnisse eine systematische Untersuchung von Artefaktbiographien erbringen kann. Denn eine analytische Unterscheidung zwischen dem zur Zeit der Entstehung eines Artefakts angedachten Publikum und seinen tatsächlichen, späteren Rezipienten fördert manch überraschende Einsicht zutage und hilft, die Inschrift nicht als statisches Objekt, sondern als dynamischen ,Aktanten' im Sinne Bruno Latours und damit als einen mit einer je singulären ,Karriere‘ versehenen Handlungsträger zu verstehen.

Die hier nur knapp umrissenen Wege für zukünftige Forschungen, welche die Aufsätze dieses Sammelbandes eröffnen, mögen als Hinweis darauf dienen, welch großes Potenzial die Untersuchung vormoderner Text-Material-Beziehungen aus der einleitend von Marc von der Höh skizzierten und im Sonderforschungsbereich 933 „Materiale Textkulturen“ verfolgten Perspektive in sich birgt. Sollte der Band dazu anregen, diese Ansätze aufzugreifen, weiterzuverfolgen und fortzuentwickeln, dann hätte er ein wichtiges Ziel erreicht. 